\title{
Molecular characterization of totiviruses in Xanthophyllomyces dendrorhous
}

\author{
Marcelo Baeza*, Natalia Bravo, Mario Sanhueza, Oriana Flores, Pablo Villarreal and Victor Cifuentes
}

\begin{abstract}
Background: Occurrence of extrachromosomal dsRNA elements has been described in the red-yeast Xanthophyllomyces dendrorhous, with numbers and sizes that are highly variable among strains with different geographical origin. The studies concerning to the encapsidation in viral-like particles and dsRNA-curing have suggested that some dsRNAs are helper viruses, while others are satellite viruses. However, the nucleotide sequences and functions of these dsRNAs are still unknown. In this work, the nucleotide sequences of four dsRNAs of the strain UCD 67-385 of $X$. dendrorhous were determined, and their identities and genome structures are proposed. Based on this molecular data, the dsRNAs of different strains of $X$. dendrorhous were analyzed.

Results: The complete sequences of L1, L2, S1 and S2 dsRNAs of $X$. dendrorhous UCD 67-385 were determined, finding two sequences for L1 dsRNA (L1A and L1B). Several ORFs were uncovered in both S1 and S2 dsRNAs, but no homologies were found for any of them when compared to the database. Instead, two ORFs were identified in each L1A, L1B and L2 dsRNAs, whose deduced amino acid sequences were homologous with a major capsid protein (5'-ORF) and a RNA-dependent RNA polymerase (3'-ORF) belonging to the Totiviridae family. The genome structures of these dsRNAs are characteristic of Totiviruses, with two overlapped ORFs (the 3'-ORF in the -1 frame with respect to the 5'-ORF), with a slippery site and a pseudoknot in the overlapped regions. These structures are essential for the synthesis of the viral polymerase as a fusion protein with the viral capsid protein through -1 ribosomal frameshifting. In the RNase protection analysis, all the dsRNAs in the four analyzed $X$. dendrorhous strains were protected from enzymatic digestion. The RT-PCR analysis revealed that, similar to strain UCD 67-385, the L1A and L1B dsRNAs coexist in the strains VKM Y-2059, UCD 67-202 and VKM Y-2786. Furthermore, determinations of the relative amounts of L1 dsRNAs using two-step RT-qPCR revealed a 40-fold increment of the ratio L1A/L1B in the S2 dsRNA-cured strain compared to its parental strain.

Conclusions: Three totiviruses, named as XdV-L1A, XdV-L1B and XdV-L2, were identified in the strain UCD 67-385 of $X$. dendrorhous. The viruses XdV-L1A and XdV-L1B were also found in other three $X$. dendrorhous strains. Our results suggest that the smaller dsRNAs (named XdRm-S1 and XdRm-S2) of strain UCD 67-385 are satellite viruses, and particularly that XdRm-S2 is a satellite of XdV-L1A.
\end{abstract}

Keywords: $X$. dendrorhous, dsRNA, Totivirus, Mycovirus

\section{Background}

Mycoviruses with double-stranded RNA (dsRNA) genomes are widespread in yeasts and filamentous fungi, and unlike mammalian viruses, they do not possess an extracellular route of infection or exhibit harmful effects against their hosts [1,2]; even if some exception have been described where the mycoviruses do have a

\footnotetext{
* Correspondence: mbaeza@uchile.cl

Departamento de Ciencias Ecológicas, Laboratorio de Genética, Facultad de Ciencias, Universidad de Chile, Las Palmeras 3425, Casilla 653, Santiago, Chile
}

deleterious effect on their hosts [3,4]. These viruses are primarily considered as functionally cryptic genetic elements, and only in a few cases, they have been associated with a detectable phenotype in the host, such as hypovirulence or antifungal activity (killer system) $[5,6]$. The dsRNA viruses encoding killer systems in Ustilago maydis and Saccharomyces cerevisiae are the best characterized [7-10], and both belong to the family Totiviridae. This family is subdivided into the genera Totivirus, Giardiavirus and Leishmaniavirus, whose members are characterized by an undivided 
dsRNA genome and isometric virions with no lipid or carbohydrate content, which are commonly denoted as Virus-Like Particles (VLPs) because they are not infectious [11]. The type species of the genus Totivirus is the $S$. cerevisiae virus L-A, found in most of the yeast strains either individually or in combination with other dsRNAs (M dsRNAs), such as occurs in the killer strains. In the 4.6-kb L-A genome, there are two overlapping ORFs: the 5'-ORF ( $\mathrm{Gag}$ ) encoding a major capsid protein (Gag or CP) and the 3'-ORF ( $\mathrm{Pol}$ ) encoding a RNA-dependent RNA polymerase (RdRp). The viral polymerase is synthesized as a fusion protein with Gag through a translational -1 frameshifting event. The genome of the M dsRNA (1.6 to $1.8 \mathrm{~kb}$ ) only has one ORF encoding a killer toxin and self-immunity, and is called a satellite virus because it depends on the proteins encoded by L-A (helper virus) for encapsidation and replication [10].

The existence of VLPs and extrachromosomal dsRNA elements with estimated lengths of 5 (L1), 3.7 (L2), 1.4 (M), 0.9 (S1) and 0.8 (S2) kbp have been described in Xanthophyllomyces dendrorhous (formerly Phaffia rhodozyma) [12-15]. This basidiomycetous yeast has been isolated from cold climate areas around the world [1620 ] and is currently one of the most promising sources of astaxanthin, a biotechnologically important carotenoid pigment [21,22]. Polymorphic dsRNA-profiles have been observed in $X$. dendrorhous in which there are strains that have zero, one, two, or four dsRNAs, and the encapsidation into VLPs have been determined for some of them $[12,13,15]$. In curing experiments performed with the UCD 67-385 strain, which has L1, L2, S1 and S2 dsRNAs, the concentration of L1 dsRNA is significantly increased as a consequence of the loss of S2 dsRNA (in the cured strain 385(S2)), while the amounts of L2 and S1 dsRNAs remain similar between the parental and cured strains $[12,13,15]$. These results suggest that the dsRNAs of $X$. dendrorhous are mycoviruses and that there exist helper/satellite systems in some strains of this yeast. However, there is no data regarding dsRNA nucleotide sequences, and the only information reported concerning relationships is derived from hybridization experiments among the dsRNAs of different $X$. dendrorhous strains, including a 5.0-kb dsRNA from Cystofilobasidium and the dsRNAs of strains CBS 5908 and ATCC 24203 of $X$. dendrorhous [23]. In this work, the molecular characterization of the dsRNA elements of $X$. dendrorhous was performed. The strain UCD 67-385 was selected for cloning and sequencing because it harbors almost all of the various dsRNAs reported in this yeast. Based on the molecular data obtained, the putative viral identity for dsRNAs was proposed, and the dsRNAs of different $X$. dendrorhous strains were analyzed using RTPCR and two-step RT-qPCR methods.

\section{Results}

\section{Cloning and sequencing of dsRNAs}

The $X$. dendrorhous strain UCD 67-385 was selected for cloning purposes because it contains two large (L1 and L2) and two small (S1 and S2) dsRNAs, representing at least in size almost all of the various dsRNAs that have been reported in different strains of this yeast [15]. All dsRNAs used for cloning purposes and RT-PCR analyses were treated with DNase I and purified from agarose gels. The SISPA methodology $[24,25]$ was used to clone the entire dsRNAs, but only positive results were obtained for small dsRNAs. Thus, an alternative strategy including the construction of cDNA libraries and primer walking was employed for the cloning of L1 and L2 dsRNAs, as described in the methods section. The cDNA sequences obtained from random clones were assembled, and contigs of approximately 1.6 and $1.5 \mathrm{kbp}$ for L1 and L2 dsRNAs, respectively, were generated. The dsRNA-origins of the cDNAs were confirmed using RT-PCR with specific primers designed from each contig. Based on these sequences, the primer-walking experiments were performed in both directions, selecting the clones in each extension step harboring larger cDNAs by colony-PCR using vector primers. Finally, the 3' ends of dsRNA molecules were determined using RLM-RACE as described in the methods section. This experimental strategy successfully generated the entire L2 dsRNA sequence, but some discrepant results were obtained for L1 dsRNA because several of the new sequences generated in the extension steps were not spliced at $100 \%$ with the preceding contigassembly. These results suggested the existence of at least two molecules of L1 dsRNA with similar lengths and different sequences. This result was confirmed through comparisons with the results obtained in the sequencing of the total RNA of $X$. dendrorhous UCD 67-385 using the sequencing-by-synthesis technology (http://www.illumina. com/technology/sequencing_technology.ilmn) on the massively parallel Illumina Genome Analyzer II. Considering the sizes and nucleotide sequences obtained, five different dsRNAs were identified: L1A (4,655 bp), L1B (4,619 bp), L2 (3,962 bp), S1 (962 bp) and S2 (766 bp), which have been deposited in the GenBank database under the numbers JN997472, JN997473, JN997474, JN997475 and JN997476, respectively.

\section{Bioinformatic analysis and genome organization of the dsRNAs}

Several ORFs with lengths ranging from 108 to $600 \mathrm{nt}$ were observed in the S1 and S2 dsRNAs (only the major ORFs are shown in Figure 1); a comparison against the NCBI database revealed no significant hits for the nucleotide sequences or translated ORFs. In all L1A, L1B and L2 dsRNAs, two ORFs (ORF1 and ORF2) were detected. The translated sequences of the 5'-ORF (ORF1) 


Figure 1 Genome structure of dsRNAs of $X$. dendrorhous UCD 67-385. The predicted ORFs and putative genes are indicated. Gag, major
capsid protein; Pol, RNA dependent RNA polymerase. The ORF-frame is indicated in parenthesis. The predicted ATG-start codons for ORF 2 are
indicated, and the length of the putative overlapping regions is denoted in the square brackets.

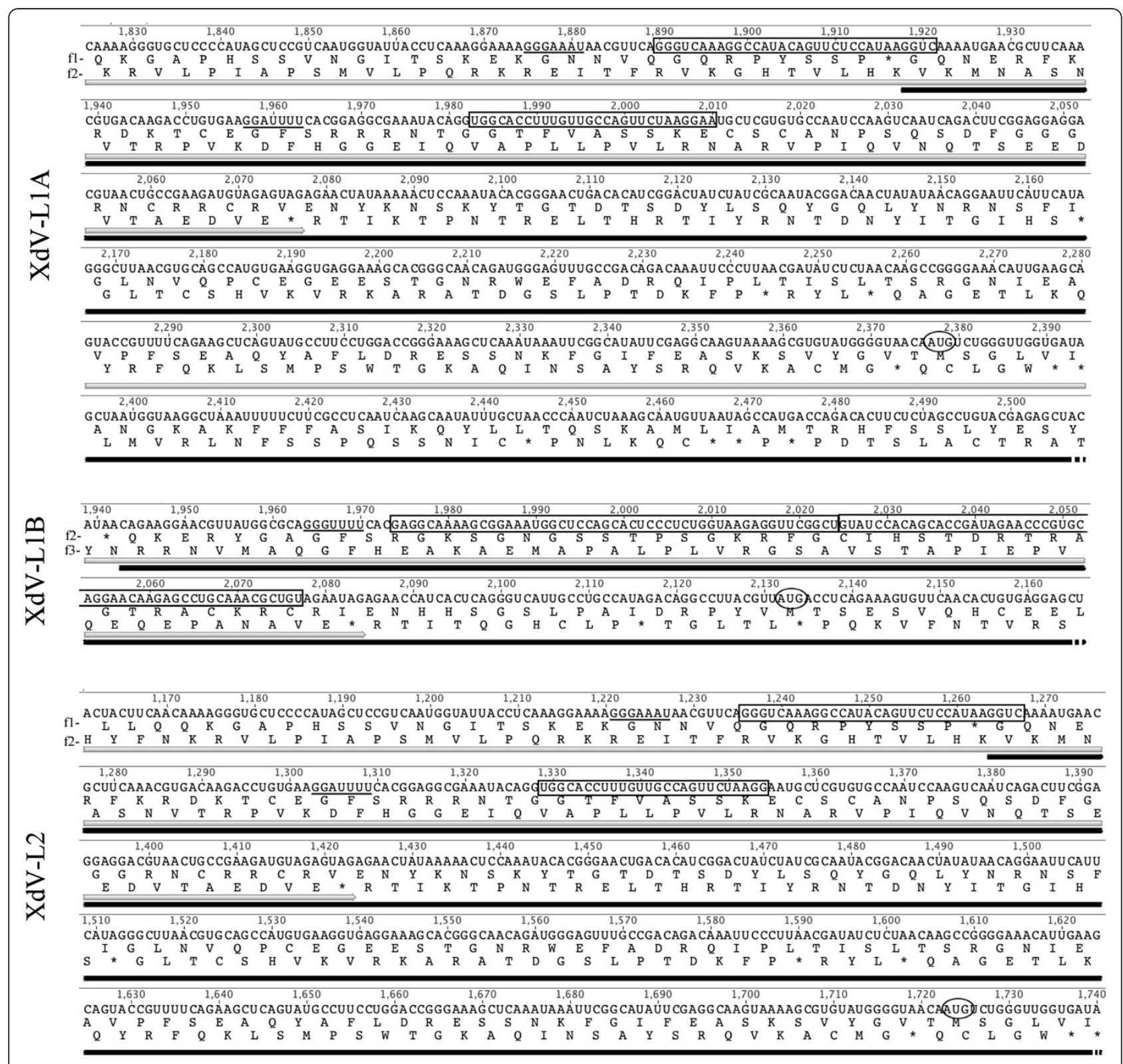

Figure 2 Overlapping regions of viral dsRNAs of $\boldsymbol{X}$. dendrorhous. The putative slippery sites are underlined. The box indicates the predicted sequences involved in the pseudoknot structures. The ORFs for the putative CP and RdRp are indicated in gray and black, respectively. 
and the 3'-ORF (ORF2) showed homologies with the major capsid protein (CP) and an RNA-dependent RNA polymerase (RdRp), respectively, of viruses belonging to the in cursive family (Figure 1). As these results strongly suggest that the dsRNAs L1A, L1B and L2 are mycoviruses, they were designated as XdV-L1A, XdV-L1B and $\mathrm{XdV}-\mathrm{L} 2$ for Xanthophyllomyces dendrorhous virus, whereas the small dsRNAs were designated as XdRm-S1 and XdRm-S2 for Xanthophyllomyces dendrorhous dsRNA molecule. The nucleotide length of the predicted 5' and 3' untranslated regions (UTRs) of each viral dsRNA are 31 and 59 nt in XdV-L1A, 32 and 25 in $\mathrm{XdV}-\mathrm{L} 1 \mathrm{~B}$ and 73 and 35 in XdV-L2, respectively. As shown in Figure 1, the ORFs for the putative RdRp ( $P o l)$ were in a -1 frame with respect to the ORFs for the putative CP $(G a g)$ in the large dsRNAs. This type of genome organization is characteristic of viruses in which the Gag and Pol genes are overlapped and the RdRp is synthesized as a fusion protein with $\mathrm{CP}$ as consequence of a ribosomal -1 frameshifting event [26,27]. Two elements in the viral (+) RNA are necessary for the efficient induction of the frameshifting event: a) a heptanucleotide called the slippery site with the sequence X XXY YYZ (triplets indicate the pre-frameshift codons) and b) a downstream stimulatory RNA pseudoknot. If the predicted ATG-start codon for $\mathrm{Pol}$ is present as internal codon, overlapping regions of 258, 189 and $258 \mathrm{nt}$ are generated in XdV-L1A, XdV-L1B and XdV-L2, respectively (Figures 1 and 2). Canonical slippery sites (X XXY YYZ) adjacent to pseudoknot structures were predicted in all three large dsRNAs (Figures 2 and 3). However, only in XdV-L1B these elements can probably induce the expression of a CP-RdRp fusion protein, as in the other two large dsRNAs both elements are located out of the overlapping region. The slippery site necessary to express the fusion proteins in XdV-LA1 and XdV-L2 can be GGAUUUU (Figures 2 and 3), a sequence slightly different from the canonical one, that can be functional as it has been demonstrated in the induction of frameshifting events in flavivirus and dianthovirus [28,29].

The comparison of the deduced amino acid sequences of RdRps of $X$. dendrorhous viruses and those of the 25 members of the in cursive family are shown in Figure 4. All eight conserved domains, which have been previously described in viral RNA polymerases [30,31], were found in the $X$. dendrorhous viruses. These domains are almost identical among $X$. dendrorhous and $S$. cerevisiae viruses, particularly in domains 5 (SGXXXTXXXNTXXXN [X = any amino acid]) and 6 (GDD), which have been demonstrated to be essential for viral function [32,33]. In the global analysis of RdRps using the neighbor-joining method, the $X$. dendrorhous viruses were grouped together with those infecting S. cerevisiae and Black raspberry (Figure 5).

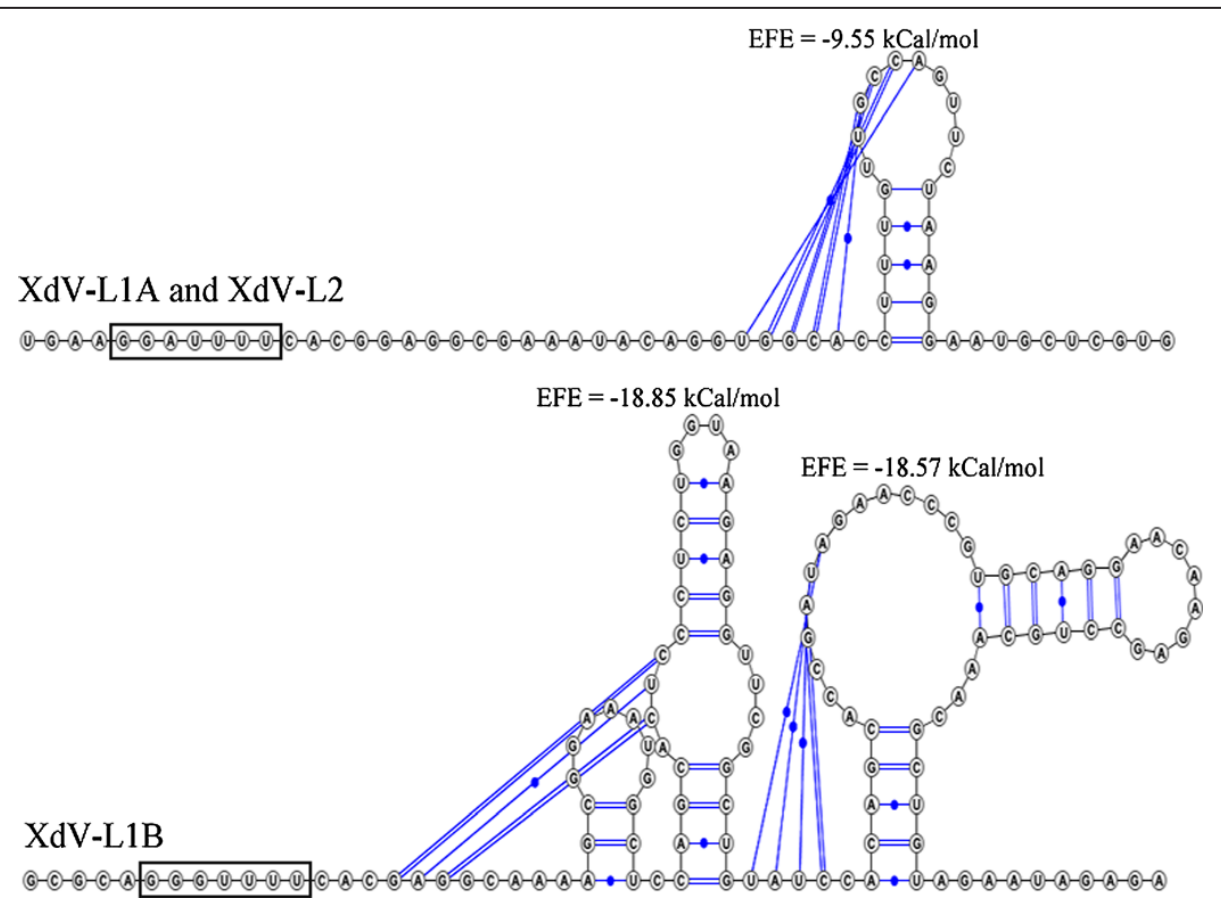

Figure 3 Pseudoknot structure prediction. The predictions were performed using a novel pseudoknot detection method called DotKnot (http://dotknot.csse.uwa.edu.au/) [56,57]. For the prediction of the estimated free energy (EFE), the method uses the pseudoknot loop entropy parameters published by Cao and Chen [58,59]. The putative slippery sites are indicated in the boxed region. 
Analysis of dsRNAs in different strains of $X$. dendrorhous

To determine if the dsRNA of others strains of $X$. dendrorhous are putative mycoviruses, the encapsidation and relation to viruses of the UCD 67-385 strain were analyzed. Samples of VLPs were extracted from cultures of each strain and subjected to RNase-protection assays. As shown in Figure 6, RNase A completely degraded the dsRNAs in the VLP samples that were deproteinized by the treatment with phenol (" $\mathrm{B}$ " lines), in contrast to the samples not treated with phenol ("A" lines); no dsRNA bands were observed in the direct analysis of the VLP samples on agarose gels (not shown), indicating that there are no free-dsRNAs that co-purified with the VLPs. Consequently, the dsRNAs observed in the assays were protected from enzymatic digestion, implying that these dsRNAs are encapsidated. Similar to the putative viral dsRNAs, the small dsRNAs are encapsidated in strains UCD 67-385 and UCD 67-202, suggesting that the S1 and S2 dsRNAs are part of the viral system infecting these strains of $X$. dendrorhous. The L1-dsRNAs in different $X$. dendrorhous strains was examined using RT-
PCR with specific primers for XdV-L1A and XdV-L1B designed in regions corresponding to the conserved domains of the RdRPs (see Table 1 and Figure 4) and the non-conserved regions of the CP-ORFs. The reversetranscription reactions were performed at $50^{\circ} \mathrm{C}$, and the amplicons of expected size were obtained from all L1dsRNAs when the conserved primers were used (Table 2), but for non-conserved primers, positive results were obtained only in L1-dsRNAs of Japanese strains (UCD 67-385 and UCD 67-202), whiles no amplicon were obtained for L1-dsRNAs of strains from Russia (VKM Y2059 and VKM Y-2786). According to these results, the L1-dsRNAs observed in the different strains of $X$. dendrorhous correspond to the two totiviruses, which are more related among strains with similar geographical origin. No amplicons were obtained from the L2 dsRNA of strain UCD 68-653C. The relative amounts of both viruses were determined using two-step RT-qPCR of the purified L1-dsRNAs with primers specific to each virus (Table 1). First, the $\mathrm{RT}$ reactions at $50^{\circ} \mathrm{C}$ were performed using the forward primers, and then the products were

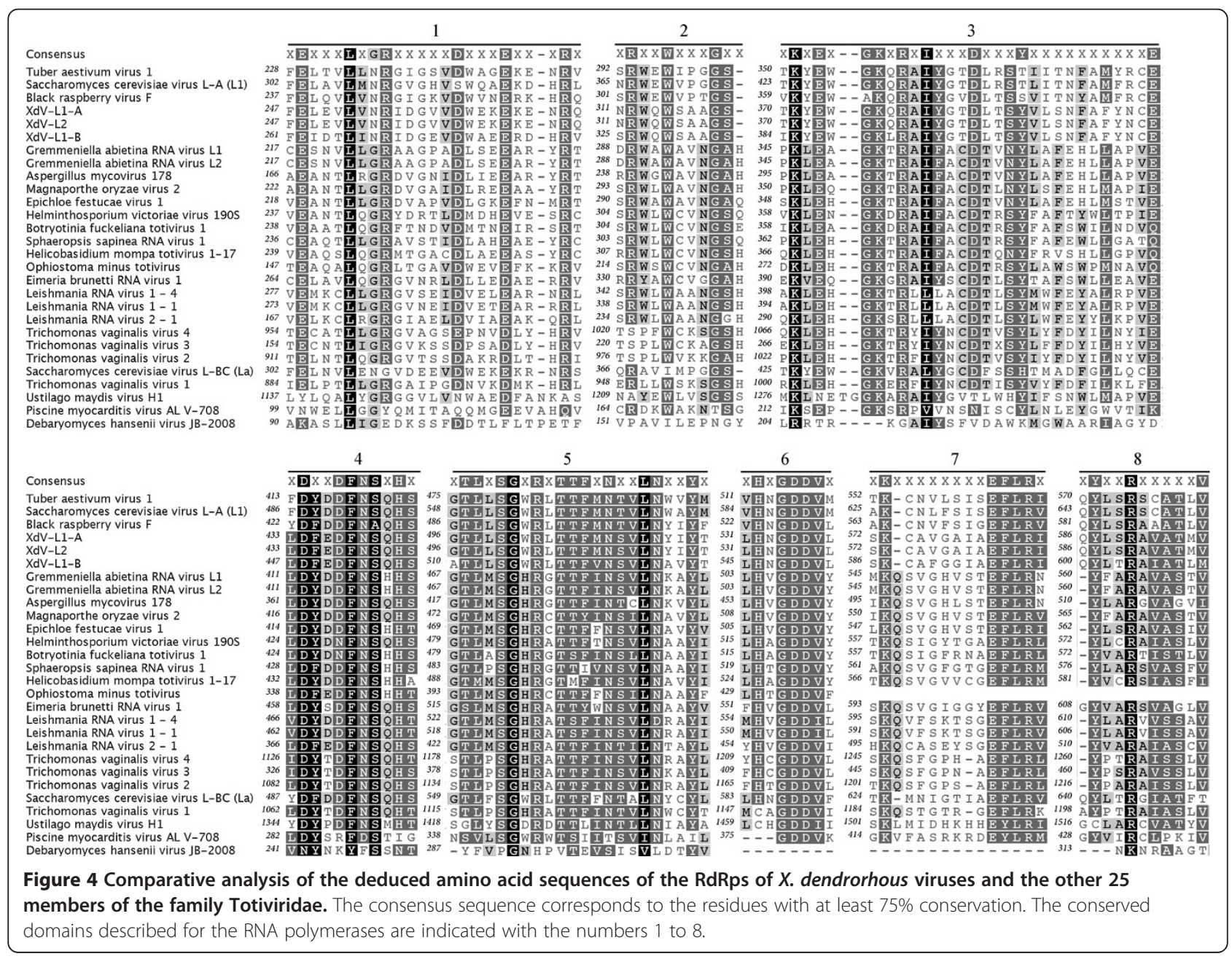




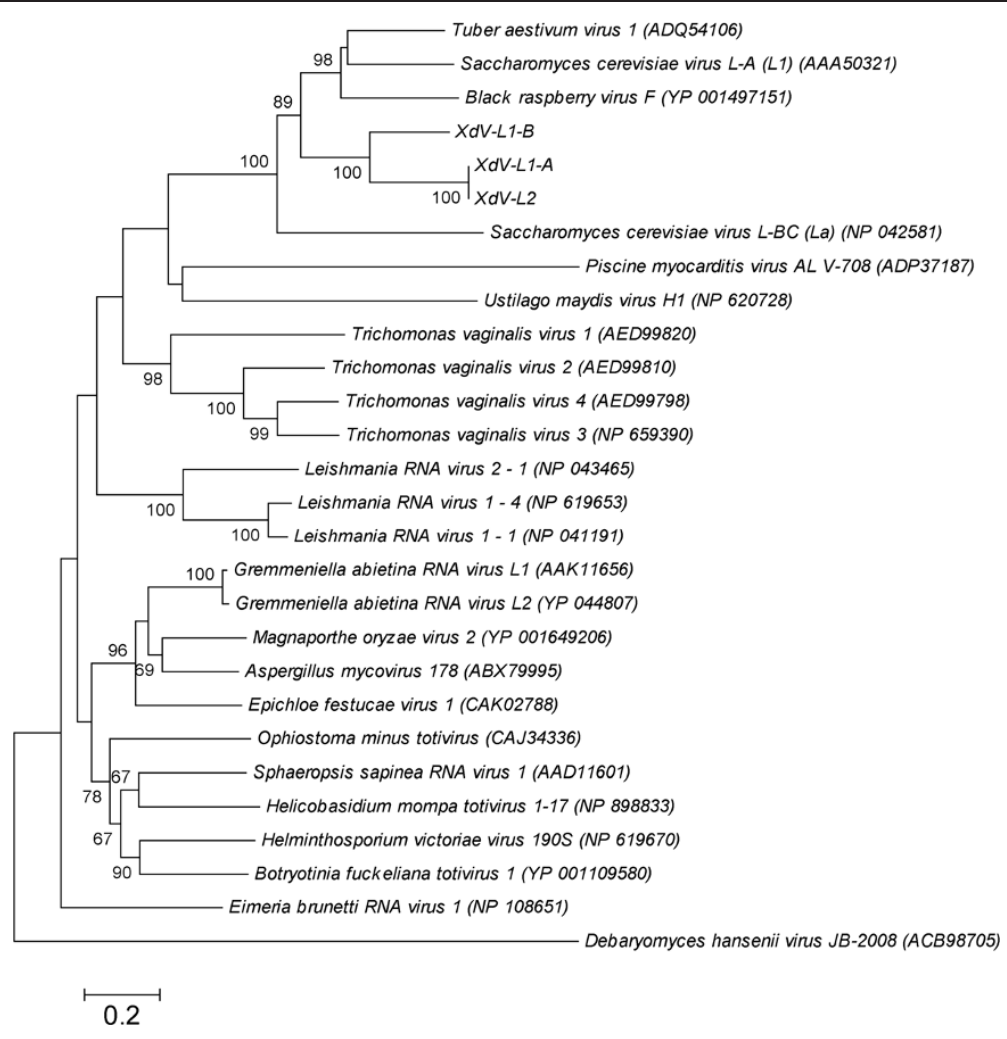

Figure 5 Unrooted neighbor-joining tree based on the RdRp amino acid sequences of $X$. dendrorhous viruses and members of the Totiviridae family. The optimal tree with the sum of branch lengths $=11.96693446$ is shown. The bootstrap values obtained for the 500 replicates are indicated next to the branches.

submitted to qPCR as described in the methods section. Several biological and technical replicates were designed to evaluate the consistency of the results. A significant amount of XdV-L1B in relation to XdV-L1A was observed in the UCD 67-385 strain, with ratio L1B/L1A of approximately 14 (Table 2). By contrast, in the cured strain 385 (S2)-40, an inversion of the viral ratio was observed with a significant amount of $\mathrm{XdV}-\mathrm{L} 1 \mathrm{~A}$ (ratio $\mathrm{L} 1 \mathrm{~B} / \mathrm{L} 1 \mathrm{~A}=0.4$; ratio $\mathrm{L} 1 \mathrm{~A} / \mathrm{L} 1 \mathrm{~B}=15$ ), suggesting an approximately 40 -fold increase in relation to its parental strain UCD 67-385.

\section{Discussion and conclusion}

Previously, it has been reported that the yeast $X$. dendrorhous strain UCD 67-385 has four extrachromosomal dsRNA elements of different lengths, called L1, L2, S1 and S2. Considering the nucleotide sequences obtained
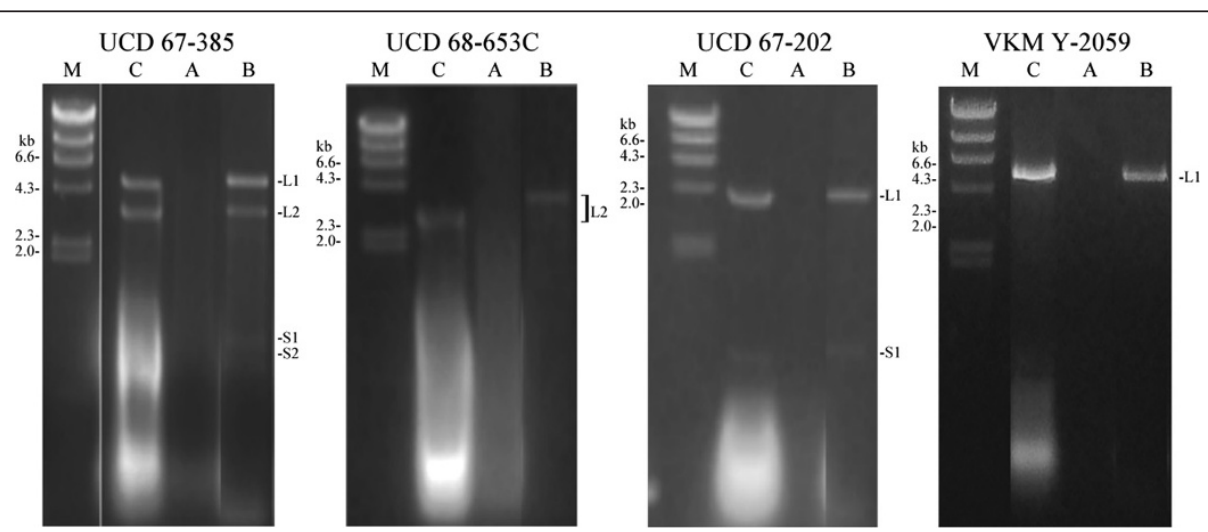

Figure 6 RNase protection assays of VLPs samples extracted from different $X$. dendrorhous strains. A: the VLPS samples digested with RNase $\left(1 \mathrm{~h}\right.$ at $\left.37^{\circ} \mathrm{C}\right)$ after phenol-chloroform extraction; B: the VLPS samples digested with RNase $\left(1 \mathrm{~h}\right.$ at $\left.37^{\circ} \mathrm{C}\right)$ before phenol-chloroform; $\mathrm{C}$ : the VLPs samples incubated for $1 \mathrm{~h}$ at $37^{\circ} \mathrm{C} ; \mathrm{M}, \lambda$ HindllI DNA marker. 
Table 1 Oligonucleotides used in this work

\begin{tabular}{|c|c|c|}
\hline Name & Position or application & Sequence $\left(5^{\prime}-3^{\prime}\right)$ \\
\hline NBA1 & Tailing of dsRNAs & (PO4)-GCAATTGTACGCCTGGAGCGC-(NH2) \\
\hline NBA-1 & Complementary to NBA1 & GCGCTCCAGGCGTACAATTGCG \\
\hline Mot4F & Conserved motif 4 of RdRp of XdV-L1A & GAGGACTTCAATAGTCAACA \\
\hline Mot5R & Conserved motif 5 of RdRp of XdV-L1A & AAGTCGTCAGCCTCCACCCC \\
\hline Mot6R & Conserved motif 6 of RdRp of XdV-L1A & AGACATCGTCTCCGTTGTGC \\
\hline L8.2R & CP ORF of XdV-L1A & CCGTGTAGAGCTAAAATACC \\
\hline SR4 & CP ORF of XdV-L1A & AAGCGCATCTTCTGGGCTCA \\
\hline LIVR3 & CP ORF of XdV-L1B & СCTCTGCTGATCTGAAATGTT \\
\hline Cap288R & CP ORF of XdV-L1B & GAAGTITCGCCCACAAGAG \\
\hline Mot4FB & Conserved motif 4 of RdRp of XdV-L1B & GAAGATTTCAACAGTCAACATAG \\
\hline Mot5RB & Conserved motif 5 of RdRp of XdV-L1B & ATGTCGTTAACCTCCAGCCC \\
\hline Mot6RB & Conserved motif 6 of RdRp of XdV-L1B & GCACGTCGTCGCCGTTATG \\
\hline L1AF & RT-qPCR, specific for XdV-L1A & GCGAAAATGAAGCGATGACA \\
\hline L1AR & RT-qPCR, specific for XdV-L1A & TTAGTCTCCGCGCCCTTCTA \\
\hline L1BF & RT-qPCR, specific for XdV-L1B & GACGAACTGATGCCCAAACA \\
\hline L1BR & RT-qPCR, specific for XdV-L1B & CCGGAGACAGCUCAUUGUA \\
\hline Random hexamer & Random cloning & NNNNNN \\
\hline M13F & Vector & GTAAAACGACGGCCAGT \\
\hline M13R & Vector & AACAGCTATGACCATG \\
\hline
\end{tabular}

F, forward; R, reverse.

in this work, there are five different dsRNAs because the L1 dsRNA corresponds to two molecules of similar lengths (not differentiable on agarose gels) and with different sequence. According to the bioinformatic analyses and comparisons against the database, the three larger dsRNAs are putative mycoviruses belonging to the family in cursive, genus in cursive, and were named XdV-L1A, $\mathrm{XdV}-\mathrm{L} 1 \mathrm{~B}$ and XdV-L2. It was observed that all of the dsRNA-harboring strains of $X$. dendrorhous are coinfected with two or three totiviruses, a phenomenon that is not uncommon in fungi; some examples of mycovirus co-infections include the potexvirus-like M-dsRNA and SsRV-L in Sclerotinia sclerotiorum strain Ep-1PN [34,35]; the totivirus Hv190SV and the chrysovirus Hv145SV in
Cochliobolus victoria [36,37]; the totiviruses SsRV-1 and SsRV-2 in Sphaeropsis sapinea [38]; the totiviruses ScVL-A and ScV-L-BC in S. cerevisiae [39]; the partitiviruses $\mathrm{S}$ and $\mathrm{F}$ in Penicillium stoloniferum [40,41]; the dsRNA1related Fusarium poae virus 1, and the dsRNA3-related Helicobasidium mompa 70 virus in the isolate V1 of the violet root rot fungus $H$. mompa [42]; the totivirus-like dsRNAs CeRV1 and CeRV2 in Chalara elegans (Thielaviopsis basicola) [43]; a mixed chrysovirus infection in a strain of the endophytic and entomopathogenic fungus Tolypocladium cylindrosporum [44]; and mycoviruses that contain 2-4 different segments of dsRNAs in Fusarium graminearum [45]. The RT-PCR analysis revealed that all L1-dsRNAs share regions encoding conserved

Table 2 RT-PCR and RT-qPCR from L1-dsRNAs isolated from different $X$. dendrorhous strains

\begin{tabular}{|c|c|c|c|c|c|c|c|c|c|}
\hline \multirow[t]{2}{*}{ Strain } & \multicolumn{8}{|c|}{ Primers pairs } & \multirow{2}{*}{$\begin{array}{l}\text { Ratio }^{a} \\
\text { L1B/L1A }\end{array}$} \\
\hline & $\begin{array}{l}\text { Mot4F } \\
\text { Mot5R }\end{array}$ & $\begin{array}{l}\text { Mot4F } \\
\text { Mot6R }\end{array}$ & $\begin{array}{l}\text { L8.2R } \\
\text { SR4 }\end{array}$ & $\begin{array}{l}\text { LIVR3 } \\
\text { Cap288Rv }\end{array}$ & $\begin{array}{l}\text { Mot4FB } \\
\text { Mot5RB }\end{array}$ & $\begin{array}{l}\text { Mot4FB } \\
\text { Mot6RB }\end{array}$ & $\begin{array}{l}\text { L1BF } \\
\text { L1BR }\end{array}$ & $\begin{array}{l}\text { L1AF } \\
\text { L1AR }\end{array}$ & \\
\hline VKM Y-2059 & + & + & - & - & + & + & - & - & nd \\
\hline UCD 67-202 & + & + & + & + & + & + & - & - & nd \\
\hline VKM Y-2786 & + & + & - & - & + & + & - & - & nd \\
\hline UCD 68-653 C* & - & - & - & - & - & - & nd & nd & nd \\
\hline UCD 67-385 & + & + & + & + & + & + & + & + & $14(6)$ \\
\hline 385(S2)-40 & + & + & + & + & + & + & + & + & $0.4(0.2)$ \\
\hline
\end{tabular}

RT-PCR determinations were made at least 3 times for each dsRNA. *, L2 dsRNA was analyzed. +: an amplicon of the expected size; -: no amplicon; nd: not determined. ${ }^{a}$, ratios were calculated from ct values obtained through RT-qPCR, the average of three biological replicates with three technical replicates are given; the standard deviations are shown in parenthesis. 
domains of the viral polymerase, but non-conserved regions are only shared by $X$. dendrorhous strains with similar geographical origin.

The three viral dsRNAs of $X$. dendrorhous have a genetic structure that is characteristic of the totiviruses, with a putative Pol gene in the -1 frame with respect to the putative Gag gene. Thus, the viral polymerase is synthesized as a fusion protein with the capsid protein through a ribosomal frame-shifting event. The two RNA elements that are necessary for the induction of this event, a slippery site and pseudoknot structure [26,27], were revealed in the overlapping regions of each larger dsRNA. This result suggests that the larger dsRNAs are functionally independent viruses in $X$. dendrorhous. The sequence of XdV-L2 is almost identical to XdV-L1A and lacks $655 \mathrm{bp}$ from the 5 '-end, suggesting the possibility that XdV-L2 is a replicative intermediate of XdV-L1A. However, we believe that XdV-L2 is an independent virus that might have derived from XdV-L1A because the differences at the ends of the genomes, it is always present independent of the growth phase of the yeast [15], it is encapsidated into VLPs (according to RNase protection assays) and it is the only stable dsRNA maintained in the $X$. dendrorhous strain UCD 68-6537C. Nevertheless, a more in-depth characterization of the VLPs in relation to the existence of different types, their genomic contents and the identification of structural proteins is necessary for a better understanding of the viral system of this yeast. Although no homologies were found for the smaller dsRNAs, these dsRNAs would be part of the viral system as satellite dsRNAs because although they do not encode viral proteins, they are encapsidated and stably maintained. This proposition is supported for XdRm-S2 because, for the strain UCD 67-385, an increase of the amount of XdV-L1 dsRNAs, but not of XdV-L2, was observed in a strain cured of $\mathrm{XdRm}-\mathrm{S} 2$ [15]; therefore, XdRm-S2 would be a satellite of XdV-L1A and/or XdV-L1B. Moreover, in the present work, we determined a specific increment of the XdVL1A in the cured strain, strongly supporting that XdRmS2 is a satellite of XdV-L1A. Contrary to the viruses of $S$. cerevisiae, the viruses of $X$. dendrorhous are extremely resistant to curing treatments. Thus, we have applied curing methodologies using different chemical agents and temperature stresses, but only the strains cured of $\mathrm{XdRm}$-S2 were obtained. Finally, there were marked differences in viral content of the strains of $X$. dendrorhous, especially from different geographical origins, with strains having no viruses and strains having multiple viruses, including complex helper/viral systems. Because dsRNA mycoviruses have no extracellular route of infection, they have evolved together with their hosts; in this way, $X$. dendrorhous is a good model for virus-host coevolution studies.

\section{Methods}

\section{Strains and culture conditions}

The strains of $X$. dendrorhous are listed in Table 3. The yeast strains were cultured at $22^{\circ} \mathrm{C}$ in YM medium $(0.3 \%$ yeast extract, $0.3 \%$ malt extract, $0.5 \%$ peptone) supplemented with $2 \%$ glucose. The cellular pellets for the extraction of dsRNA or VLPs were obtained after the centrifugation at 7,000 $\mathrm{xg}$ for $10 \mathrm{~min}$ of cultures in the late exponential phase of growth. E. coli was grown at $37^{\circ} \mathrm{C}$ in $\mathrm{LB}$ medium ( $1 \%$ tryptone, $0.5 \%$ yeast extract, $0.5 \% \mathrm{NaCl}$ ) supplemented with $0.1 \%$ glucose. For semisolid media, 1.5\% agar (Oxoid) was used. When required, ampicillin and X-Gal were added to the media at 100 and $75 \mu \mathrm{g} / \mathrm{ml}$, respectively.

\section{The extraction of VLPs and the RNase protection assay}

The yeast pellet (4-6 g) was washed once with distilled water and suspended in $20 \mathrm{ml}$ of TBS buffer $(10 \mathrm{mM}$ Tris, $100 \mathrm{mM} \mathrm{NaCl}, 1 \mathrm{mM} \mathrm{MgCl} 2,0.1 \mathrm{mM}$ EDTA, $\mathrm{pH}$ 7.4) supplemented with "CompleteTM Protease Inhibitor Cocktail Tablets" (Roche) according to the manufacturer's instructions. Three grams of glass beads $(0.5 \mathrm{~mm}$ in diameter) were added, and the samples were shaken for $30 \mathrm{~s}$ in a Mini beadbeater-16 homogenizer (Bio Spec, Bartlesville, USA), and subsequently cooled on ice for $1 \mathrm{~min}$; the shaking and cooling steps were repeated four times. The supernatant was harvested after centrifugation at $12,000 \mathrm{xg}$ for $20 \mathrm{~min}$ and subjected to an additional centrifugation step at 120,000 xg for $90 \mathrm{~min}$ (both

\section{Table $3 X$. dendrorhous strains used in this work}

\begin{tabular}{lll}
\hline Strains & Source & dsRNAs [15] \\
\hline VKM Y-2059 & Flux of Betula verrucosa, Moscow Region, Russia. & L1 \\
UCD 68-653C & Exudate of Betula papyrifera, Rainbow Lake, Kenai Peninsula, AK. & L2 \\
UCD 67-385 & Exudate of Betula tauschii, Shinkai, Kiso, Japan. & L1, L2, S1 and S2 \\
UCD 67-202 & Cornus brachypoda, Hiroshima, Japan. & L1 and S1 \\
VKM Y-2786 & Exudate of Betula verrucosa, Moscow Region, Russia. & L1 \\
$385(S 2)-40$ & S2 dsRNA-cured strain from UCD 67-385. & L1, L2 and S1 \\
\hline
\end{tabular}


centrifugations were performed at $4^{\circ} \mathrm{C}$ ), and the pellet, enriched in VLPs, was suspended in $1.5 \mathrm{ml}$ of TBS buffer. For the RNase protection assays, the samples were digested with $50 \mu \mathrm{g} / \mathrm{ml}$ RNase A for $30 \mathrm{~min}$ at $37^{\circ} \mathrm{C}$, before and after the organic extraction with 1 volume of phenol ( $\mathrm{pH} 4.0)$ and chloroform: isoamylic alcohol (24:1). The samples were analyzed using agarose gel electrophoresis.

\section{Purification of dsRNA molecules}

The yeast pellet $(0.1 \mathrm{~g})$ was washed twice with $1 \mathrm{ml}$ of TE buffer (10 mM Tris-HCl, 1 mM EDTA, $\mathrm{pH}$ 8.0) and suspended in $0.4 \mathrm{ml}$ of the same buffer. Four hundred microliters of $0.5 \mathrm{~mm}$-diameter glass beads and $400 \mu \mathrm{l}$ of acid phenol (equilibrated with $50 \mathrm{mM}$ sodium acetate, $\mathrm{pH}$ 4.0) were added, and the tubes were shaken for $3 \mathrm{~min}$ in a Mini beadbeater-16 homogenizer. The samples were centrifuged at $10,000 \mathrm{xg}$ for $2 \mathrm{~min}$, and the aqueous phase was extracted once with $0.4 \mathrm{ml}$ of saturated acid phenol and twice with $0.4 \mathrm{ml}$ of chloroform: isoamyl alcohol (24:1). The aqueous phase was transferred to an Eppendorf tube, and two volumes of isopropanol were added. The mixture was incubated at $-20^{\circ} \mathrm{C}$ for 1 to $2 \mathrm{~h}$. The RNAs were obtained after centrifugation at 14,000 $\mathrm{xg}$ for $20 \mathrm{~min}$, dried and suspended in 20-40 $\mu \mathrm{l}$ of nuclease-free water. The samples were digested with DNase I for $1 \mathrm{~h}$ at $37^{\circ} \mathrm{C}$ and analyzed using $1 \%$ agarose gel electrophoresis in TAE buffer ( $2 \mathrm{M}$ Tris base, 1.6 M glacial acetic acid, and $0.05 \mathrm{M}$ EDTA, pH 8.0). The gels were stained with ethidium bromide $(0.5 \mu \mathrm{g} / \mathrm{ml})$, and each band corresponding to a dsRNA molecule was purified from the gels as previously described [46].

\section{Cloning of dsRNAs using the Sequence-Independent Single-Primer Amplification (SISPA) methodology}

Gel-purified dsRNAs (300 ng) were ligated to NBA1 primer (250 ng) using $2 \mathrm{U}$ of T4 DNA ligase and $10 \mathrm{U}$ of T4 RNA ligase 2 at $37^{\circ} \mathrm{C}$ for $16 \mathrm{~h}$. The primer-tailed dsRNAs were purified using the UltraClean ${ }^{\text {"m }} 15$ DNA purification kit (MO BIO Laboratories), denatured at $95^{\circ} \mathrm{C}$ for $10 \mathrm{~min}$ in the presence of $20 \%$ DMSO and $5 \mathrm{ng}$ of the NBA-1 primer, and subsequently chilled in an ice water bath. The cDNAs were synthesized with $200 \mathrm{U}$ of Maxi$\mathrm{ma}{ }^{\circledR}$ Reverse Transcriptase (Fermentas International, Inc.) at $55^{\circ} \mathrm{C}$ for $90 \mathrm{~min}$. After digestion with RNase $\mathrm{H}$ at $37^{\circ} \mathrm{C}$ for $10 \mathrm{~min}$, the partial duplexes were filled in using Taq DNA polymerase at $72^{\circ} \mathrm{C}$ for $5 \mathrm{~min}$. The PCR reactions were performed using the NBA-1 primer and the amplicons obtained were separated and purified from gels. These products were cloned using the Zero Blunt ${ }^{\circledR}$ $\mathrm{TOPO}^{\circledR}$ PCR Cloning Kit (Invitrogen). The recombinant clones were selected using colony-PCR and the M13F and M13R primers.

\section{Cloning of dsRNAs through cDNA library construction and primer walking}

A mixture of gel-purified dsRNAs (200-300 ng) and random hexanucleotide primers (200 ng) was incubated at $95^{\circ} \mathrm{C}$ for $10 \mathrm{~min}$ in the presence of $20 \%$ DMSO, chilled in an ice water bath and incubated at $25^{\circ} \mathrm{C}$ for $10 \mathrm{~min}$. The denatured dsRNAs were reverse transcribed with $200 \mathrm{U}$ of M-MulV Reverse Transcriptase (New England Biolabs) at $42^{\circ} \mathrm{C}$ for $90 \mathrm{~min}$, followed by digestion with $4 \mathrm{U}$ of RNase $\mathrm{H}$ at $37^{\circ} \mathrm{C}$ for $90 \mathrm{~min}$. The second cDNA strand was synthesized using $50 \mathrm{U}$ of DNApol I (New England Biolabs) at $37^{\circ} \mathrm{C}$ for $120 \mathrm{~min}$, followed by treatments with $2 \mathrm{U}$ of T4-DNA ligase at $22^{\circ} \mathrm{C}$ for $30 \mathrm{~min}$ and $5 \mathrm{U}$ of Klenow fragment at $37^{\circ} \mathrm{C}$ for $60 \mathrm{~min}$. The cDNAs were purified on Sephadex G-50 columns (GE Healthcare), mixed with $E c o R V$-digested pBluescript SK plasmid and $2 \mathrm{U}$ of T4-DNA ligase, incubated at $15^{\circ} \mathrm{C}$ for $16 \mathrm{~h}$, and dialyzed through a $0.025-\mu \mathrm{m}$ pore nitrocellulose disk (Millipore). The ligation mixture was used to transform E. coli DH5a cells using electroporation, and the white colonies that developed on the LB-amp-XGal plates were analyzed by colony-PCR with the vector primers M13F and M13R. The clones showing an amplicon of at least $200 \mathrm{bp}$ were selected for plasmid purification and sequencing, and the cDNA sequences obtained were analyzed and assembled. To extend the contig-sequence obtained, divergent primers were designed at the ends of the assembly and used for dsRNA reverse-transcription; the cloning and selection of clones bearing a larger cDNA insert were performed as described above. The new obtained sequences were spliced with the preceding contig-assembly; this "extension step" was repeated until the entire dsRNA was nearly cloned. The 3'-ends of the dsRNAs were cloned using the RNA ligase-mediated amplification of cDNA ends (RLM-RACE) method [47,48]. Briefly, the oligonucleotide NBA1 was ligated to the dsRNA using a T4 RNA ligase, and the primer-tailed dsRNAs were purified using the UltraClean 15 DNA purification kit. The RT-PCR reactions were performed using the primer NBA-1 and specific internal primers for dsRNAs, and the amplicons were purified and sequenced.

\section{Two-step RT-qPCR}

A mixture of $10 \mathrm{ng}$ of dsRNA, $2 \mu \mathrm{l}$ of DMSO and $1 \mu \mathrm{l}$ of the forward primer $(25 \mu \mathrm{M})$ was incubated at $94^{\circ} \mathrm{C}$ for $10 \mathrm{~min}$. Subsequently, $1 \mu \mathrm{l}$ of dNTPs $(25 \mathrm{mM}), 200 \mathrm{U}$ of Maxima Reverse Transcriptase (Fermentas Life Sciences), $4 \mu \mathrm{l}$ of $5 \mathrm{X}$ RT buffer and $9 \mu \mathrm{l}$ of nuclease-free water were added. The mixture was incubated at $50^{\circ} \mathrm{C}$ for $90 \mathrm{~min}$, followed by an additional incubation at $85^{\circ} \mathrm{C}$ for $5 \mathrm{~min}$. The qPCR was performed in a MX3000P Real-time PCR Thermal cycler (Stratagene) using the 2X Sensimix SYBR kit (Bioline) according to the following conditions: volumes of $0.5,1$ or $3 \mu \mathrm{l}$ of RT reaction products, $10 \mu \mathrm{l}$ 
of Sensimix Kit, $1 \mu \mathrm{l}$ primers mix $(50 \mu \mathrm{M}$ each), and nuclease-free water to a $20 \mu \mathrm{l}$ final volume. The PCR cycling parameters were programmed as follows: incubation at $95^{\circ} \mathrm{C}$ for $10 \mathrm{~min}$, followed by 40 cycles at $95^{\circ} \mathrm{C}$ for $15 \mathrm{~s}$, $60^{\circ} \mathrm{C}$ for $15 \mathrm{~s}$ and $72^{\circ} \mathrm{C}$ for $15 \mathrm{~s}$, and at $95,25,70$ and $95^{\circ} \mathrm{C}$ for 10, 5, 1 and $1 \mathrm{~s}$, respectively. The Mxpro-Mx300P v3.20 software (Stratagene) was used to calculate the $\mathrm{Ct}$ values, and relative quantifications were made using the $2^{-\Delta \Delta C T}$ method $[49,50]$.

\section{Automated DNA sequencing and data analysis}

The nucleotide sequences were determined using the DNA Sequencing Kit Dynamic Termination Cycle (Amersham Biosciences Limited) and the Genetic analyzer 3100 Avant automatic sequencer (Applied Biosystem). The Macrogen INC. sequencing service was also used. The sequence data were analyzed using the Geneious Pro 5.4.5 software (Biomatters, Auckland, New Zealand).

\section{Phylogenetic analysis}

The available amino acid sequences of the RdRp of totiviruses were retrieved from GeneBank and aligned together according to the deduced amino acid sequences of the RdRp of $X$. dendrorhous viruses employing Clustal W [51]. The evolutionary analyses were conducted using MEGA5 software [52] and the Neighbor-Joining method [53], and the evolutionary distances were computed using the Poisson correction method [54]. A bootstrapping of 500 replications was used to evaluate the robustness of the branching [55]. All positions containing gaps and missing data were eliminated.

\section{Competing interests}

The authors declare that they have no competing interests.

\section{Authors' contributions}

NB and MS, carried out the cloning and sequencing procedures and data analysis; OF, performed the RNase protection assays and RT-PCR experiments; $P V$, performed RT-PCR and RT-qPCR experiments. VC, participated in the study design; $\mathrm{MB}$ conceived the study and participated in its design and coordination; and MB and VC wrote the manuscript. All authors approved the final manuscript

\section{Acknowledgements}

This work was supported by grants Fondecyt 11060157 and 1100324

Received: 7 December 2011 Accepted: 17 July 2012

Published: 28 July 2012

\section{References}

1. Ghabrial SA: Origin, adaptation and evolutionary pathways of fungal viruses. Virus Genes 1998, 16:119-131.

2. Ghabrial SA, Suzuki N: Viruses of plant pathogenic fungi. Annu Rev Phytopathol 2009, 47:353-384.

3. Bhatti MF, Jamal A, Petrou MA, Cairns TC, Bignell EM, Coutts RH: The effects of dsRNA mycoviruses on growth and murine virulence of Aspergillus fumigatus. Fungal Genet Biol 2011

4. van Diepeningen AD, Debets AJ, Hoekstra RF: Dynamics of dsRNA mycoviruses in black Aspergillus populations. Fungal Genet Biol 2006, 43:446-452.
5. Pearson MN, Beever RE, Boine B, Arthur K: Mycoviruses of filamentous fungi and their relevance to plant pathology. Mol Plant Pathol 2009, 10:115-128

6. Magliani W, Conti S, Gerloni M, Bertolotti D, Polonelli L: Yeast killer systems. Clin Microbiol Rev 1997, 10:369-400.

7. Bruenn J: The double-stranded RNA viruses of Ustilago maydis and their killer toxins. dsRNA genetic elements: concepts and applications in agriculture, forestry, and medicine. Florida: CRC Press Boca Raton; 2002:109-124.

8. Kang J, Wu J, Bruenn JA, Park C: The H1 double-stranded RNA genome of Ustilago maydis virus-H1 encodes a polyprotein that contains structural motifs for capsid polypeptide, papain-like protease, and RNA-dependent RNA polymerase. Virus Res 2001, 76:183-189.

9. Schmitt MJ, Breinig F: Yeast viral killer toxins: lethality and self-protection. Nat Rev Microbiol 2006, 4:212-221.

10. Tipper DJ, Schmitt MJ: Yeast dsRNA viruses: replication and killer phenotypes. Mol Microbiol 1991, 5:2331-2338.

11. Wickner RB, Wang CC, Patterson JL: Family Totiviridae. In Virus taxonomy: classification and nomenclature of viruses: eighth report of the International Committee on the Taxonomy of Viruses. Edited by Fauquet CM, Mayo MA, Maniloff J, Desselberger U, Ball LA. San Diego, CA: Elsevier Academic Press; 2005:571-580

12. Castillo A, Cifuentes V: Presence of double-stranded RNA and virus-like particles in Phaffia rhodozyma. Curr Genet 1994, 26:364-368.

13. Pfeiffer I, Kucsera J, Varga J, Parducz A, Ferenczy L: Variability and inheritance of double-stranded RNA viruses in Phaffia rhodozyma. Curr Genet 1996, 30:294-297.

14. Pfeiffer I, Litter J, Kucsera J: Effects of double-stranded rna viruses on the reproduction of Phaffia rhodozyma. Acta Biol Hung 2001, 52:299-306.

15. Baeza M, Sanhueza M, Flores O, Oviedo V, Libkind D, Cifuentes V: Polymorphism of viral dsRNA in Xanthophyllomyces dendrorhous strains isolated from different geographic areas. Virol J 2009, 6:160.

16. Miller MW, Yoneyama MMS: Phaffia, a New Yeast Genus in the Deuteromycotina (Blastomycetes). Int I Syst Bacteriol 1976, 26:6.

17. Golubev WI: Perfect state of Rhodomyces dendrorhous (Phaffia rhodozyma). Yeast 1995, 11:101-110.

18. Weber RWS, Davoli P, Anke H: A microbial consortium involving the astaxanthin producer Xanthophyllomyces dendrorhous on freshly cut birch stumps in Germany. Mycologist 2006, 20:57-61.

19. Libkind D, Moline M, de Garcia V, Fontenla S, van Broock M: Characterization of a novel South American population of the astaxanthin producing yeast Xanthophyllomyces dendrorhous (Phaffia rhodozyma). J Ind Microbiol Biotechnol 2008, 35:151-158.

20. Libkind D, Ruffini A, van Broock M, Alves L, Sampaio JP: Biogeography, host specificity, and molecular phylogeny of the basidiomycetous yeast Phaffia rhodozyma and its sexual form, Xanthophyllomyces dendrorhous. Appl Environ Microbiol 2007, 73:1 120-1125.

21. Yuan JP, Peng J, Yin K, Wang JH: Potential health-promoting effects of astaxanthin: A high-value carotenoid mostly from microalgae. Mol Nutr Food Res 2011, 55:150-165.

22. Higuera-Ciapara I, Felix-Valenzuela L, Goycoolea FM: Astaxanthin: a review of its chemistry and applications. Crit Rev Food Sci Nutr 2006, 46:185-196.

23. Pfeiffer I, Golubev WI, Kucsera J, Golubev N: Mycocin production in Cryptococcus aquaticus. Antonie Van Leeuwenhoek 2004, 369-375.

24. Lambden PR, Cooke SJ, Caul EO, Clarke IN: Cloning of noncultivatable human rotavirus by single primer amplification. J Virol 1992, 66:1817-1822.

25. Potgieter AC, Steele AD, van Dijk AA: Cloning of complete genome sets of six dsRNA viruses using an improved cloning method for large dsRNA genes. J Gen Virol 2002, 83:2215-2223.

26. Brierley I, Gilbert RJC, Pennell S: In Pseudoknot-Dependent Programmed ?1 Ribosomal Frameshifting: Structures, Mechanisms and Models. Edited by Atkins JF, Gesteland RF. New York, NY: Springer New York; 2010:149-174.

27. Ten Dam EB, Pleij CW, Bosch L: RNA pseudoknots: Translational frameshifting and readthrough on viral RNAs. Virus Genes 1990, 4:121.

28. Firth AE, Blitvich BJ, Wills NM, Miller CL, Atkins JF: Evidence for ribosomal frameshifting and a novel overlapping gene in the genomes of insectspecific flaviviruses. Virology 2010, 399:153-166.

29. Kim KH, Lommel SA: Identification and analysis of the site of -1 ribosomal frameshifting in red clover necrotic mosaic virus. Virology 1994, 200:574-582.

30. Bruenn JA: A structural and primary sequence comparison of the viral RNA-dependent RNA polymerases. Nucleic Acids Res 2003, 31:1821-1829. 
31. Koonin EV, Choi GH, Nuss DL, Shapira R, Carrington JC: Evidence for common ancestry of a chestnut blight hypovirulence-associated doublestranded RNA and a group of positive-strand RNA plant viruses. Proc Natl Acad Sci USA 1991, 88:10647-10651.

32. Ribas JC, Wickner RB: RNA-dependent RNA polymerase consensus sequence of the LA double-stranded RNA virus: definition of essential domains. Proc Natl Acad Sci USA 1992, 89:2185.

33. Routhier $E$, Bruenn JA: Functions of conserved motifs in the RNAdependent RNA polymerase of a yeast double-stranded RNA virus. J Virol 1998, 72:4427

34. Xie J, Wei D, Jiang D, Fu Y, Li G, Ghabrial S, Peng Y: Characterization of debilitation-associated mycovirus infecting the plant-pathogenic fungus Sclerotinia sclerotiorum. J Gen Virol 2006, 87:241-249.

35. Liu H, Fu Y, Jiang D, Li G, Xie J, Peng Y, Yi X, Ghabrial SA: A novel mycovirus that is related to the human pathogen hepatitis $E$ virus and rubi-like viruses. J Virol 2009, 83:1981-1991.

36. Ghabrial SA: A transmissible disease of Helminthosporium victoriae: Evidence for a viral etiology. Boca Raton, FL: CRC Press; 1986:163-176.

37. Ghabrial SA, Soldevila Al, Havens WM: Molecular genetics of the viruses infecting the plant pathogenic fungus Helminthosporium victoriae. Molecular Biology of Double-stranded RNA: Concepts and Applications in Agriculture, Forestry and Medicine 2002, 213-236.

38. Preisig $\mathrm{O}$, Wingfield $\mathrm{BD}$, Wingfield $\mathrm{MJ}$ : Coinfection of a fungal pathogen by two distinct double-stranded RNA viruses. Virology 1998, 252:399-406.

39. Wickner R, Ribas J, Searfoss A: The double-stranded RNA viruses of Saccharomyces cerevisiae. In dsRNA genetic elements. Concepts and applicaiotns in agriculture, forestry, and medicine. Edited by SM T. Boca Raton: CRC Press; 2002:67-108.

40. Kim JW, Choi EY, Lee Jl: Genome organization and expression of the Penicillium stoloniferum virus F. Virus Genes 2005, 31:175-183.

41. Kim JW, Kim SY, Kim KM: Genome organization and expression of the Penicillium stoloniferum virus S. Virus Genes 2003, 27:249-256.

42. Osaki H, Nomura K, Matsumoto N, Ohtsu Y: Characterization of doublestranded RNA elements in the violet root rot fungus Helicobasidium mompa. Mycol Res 2004, 108:635-640.

43. Park Y, James D, Punja ZK: Co-infection by two distinct totivirus-like double-stranded RNA elements in Chalara elegans (Thielaviopsis basicola). Virus Res 2005, 109:71-85.

44. Herrero N, Zabalgogeazcoa I: Mycoviruses infecting the endophytic and entomopathogenic fungus Tolypocladium cylindrosporum. Virus Res 2011, 160:409-413.

45. Chu YM, Lim WS, Yea SJ, Cho JD, Lee YW, Kim KH: Complexity of dsRNA mycovirus isolated from Fusarium graminearum. Virus Genes 2004, 28:135-143.

46. Boyle JS, Lew AM: An inexpensive alternative to glassmilk for DNA purification. Trends Genet 1995, 11:8.

47. Coutts R, Livieratos C: A Rapid Method for Sequencing the 5'- and 3'-Termini of Double-Stranded RNA Viral Templates using RLM-RACE. J Phytopathology 2003, 527:525-527.

48. Liu X, Gorovsky MA: Mapping the $5^{\prime}$ and 3 ' ends of Tetrahymena thermophila mRNAs using RNA ligase mediated amplification of CDNA ends (RLM-RACE). Nucleic Acids Res 1993, 21:4954.

49. Pfaffl MW: A new mathematical model for relative quantification in realtime RT-PCR. Nucleic Acids Res 2001, 29:e45.

50. Livak KJ, Schmittgen TD: Analysis of relative gene expression data using real-time quantitative PCR and the $2^{-{ }^{\Delta}}{ }^{\Delta}$ CT method. Methods 2001, 25:402-408

51. Brunt J, Webb MD, Peck MW: Rapid affinity immunochromatography column-based tests for sensitive detection of Clostridium botulinum neurotoxins and Escherichia coli 0157. Appl Environ Microbiol 2010, 76:4143-4150.

52. Tamura K, Peterson D, Peterson N, Stecher G, Nei M, Kumar S: MEGA5: molecular evolutionary genetics analysis using maximum likelihood, evolutionary distance, and maximum parsimony methods. Mol Biol Evol 2011, 28:2731-2739.

53. Saitou N, Nei M: The neighbor-joining method: a new method for reconstructing phylogenetic trees. Mol Biol Evol 1987, 4:406-425.

54. Zuckerkandl E, Pauling L: Evolutionary divergence and convergence in proteins. Evolving genes and proteins 1965, 97:166.

55. Felsenstein J: Confidence limits on phylogenies: an approach using the bootstrap. Evolution 1985, 39:783-791.
56. Sperschneider J, Datta A: DotKnot: pseudoknot prediction using the probability dot plot under a refined energy model. Nucleic Acids Res 2010, 38:e103.

57. Sperschneider J, Datta A, Wise MJ: Heuristic RNA pseudoknot prediction including intramolecular kissing hairpins. RNA 2011, 17:27-38.

58. Cao S, Chen SJ: Predicting structures and stabilities for H-type pseudoknots with interhelix loops. RNA 2009, 15:696-706.

59. Cao S, Chen SJ: Predicting RNA pseudoknot folding thermodynamics. Nucleic Acids Res 2006, 34:2634-2652.

doi:10.1186/1743-422X-9-140

Cite this article as: Baeza et al.: Molecular characterization of totiviruses

in Xanthophyllomyces dendrorhous. Virology Journal 2012 9:140.

\section{Submit your next manuscript to BioMed Central and take full advantage of:}

- Convenient online submission

- Thorough peer review

- No space constraints or color figure charges

- Immediate publication on acceptance

- Inclusion in PubMed, CAS, Scopus and Google Scholar

- Research which is freely available for redistribution

Submit your manuscript at www.biomedcentral.com/submit
C Biomed Central 